Filomat xx (yyyy), zzz-zzz

DOI (will be added later)
Published by Faculty of Sciences and Mathematics, University of Niš, Serbia

Available at: http://www.pmf.ni.ac.rs/filomat

\title{
Lorentzian isotropic Lagrangian immersions
}

\author{
Franki Dillen ${ }^{\mathrm{a}}$, Luc Vrancken ${ }^{\mathrm{b}}$ \\ ${ }^{a}$ Katholieke Universiteit Leuven, Departement Wiskunde, BE-3001 Leuven, Belgium. \\ ${ }^{b}$ Univ. Lille Nord de France, F-59000 Lille, France; \\ UVHC, LAMAV, F-59313 Valenciennes, France; \\ Katholieke Universiteit Leuven, Departement Wiskunde, BE-3001 Leuven, Belgium.
}

\begin{abstract}
In this note we are interested in isotropic totally real Lorentzian submanifolds of indefinite complex space forms. We show that such submanifolds are always $H$-umbilical warped product immersions and we determine also the warping function.
\end{abstract}

Key words: Lagrangian submanifold, complex projective space, warped product, isotropic submanifold, Lorentzian submanifold.

Subject class: 53B25, 53B20.

\section{Introduction}

The notion of isotropic submanifold was first introduced in [8] by O'Neill for immersions of Riemannian manifolds and recently extended by Cabrerizo, Fernandez and Gomez in [2] to the pseudo-Riemannian case. A submanifold is called isotropic if and only if for any point $p$ and any tangent vector $X$ at a point $p$, we have that

$$
\langle h(X, X), h(X, X)\rangle=\lambda(p)\langle X, X\rangle^{2}
$$

where $h$ denotes the second fundamental form of the immersion and $\lambda$ is a function on the submanifold.

Here we will study Lagrangian isotropic immersions of a Lorentzian manifold into a complex Lorentzian space form. The similar problem in the Riemannian case is completely solved in [6], [11] and [5]. The starting ingredients in the proof in the Riemannian case is the fact that the unit sphere in the tangent space at a point is compact. Of course in the Lorentzian case it is not possible to follow the same procedure. One might expect the argument as well as the result to be more complicated but this is not the case. Where in the Riemannian case some special immersions of locally symmetric spaces appear, in the Lorentzian case we show the following:

Theorem 1.1. Let Mbe a Lagrangian Lorentzian isotropic submanifold of a complex space form then $M$ is $H$-umbilical.

2010 Mathematics Subject Classification. Primary 53B25; Secondary 53B20

Keywords. Lagrangian submanifold, complex projective space, warped product, isotropic submanifold, Lorentzian submanifold Received: dd Month yyyy; Accepted: dd Month yyyy

Communicated by Ljubica Velimirovic

Email address: luc.vrancken@univ-valenciennes.fr (Luc Vrancken) 
The notion of an $H$-umbilical Lagrangian submanifold was first introduced by Chen in [3] who in [4] also gave a classification of the $H$ umbilical submanifolds in indefinite complex Euclidean spaces. From that classification, the isotropic immersions can be determined. For that reason, in the last part of the paper, we restrict ourselves to the case that the holomorphic sectional curvature is nonvanishing and we describe the isotropic Lagrangian immersions explicitly.

\section{Preliminaries}

Throughout this paper we will assume that $M$ is a Lagrangian Lorentzian submanifold $M$ of a complex space form $\tilde{M}$. We use the standard formulas of Gauss and Weingarten for a submanifold. Thus introducing the second fundamental form $h$ and the shape operators $A$ by

$$
\begin{aligned}
& \tilde{\nabla}_{X} Y=\nabla_{X} Y+h(X, Y) \\
& \tilde{\nabla}_{X} \xi=-A_{\xi} X+\nabla_{X}^{\perp} \xi .
\end{aligned}
$$

Here as usual $\tilde{\nabla}$ denotes the Levi Civita connection on the ambient space and if no confusion is possible we will always identify $M$ with its image in $\tilde{M}$.

As $M$ is Lagrangian we have that the complex structure $J$ interchanges the tangent and the normal space. Using the formulas of Gauss and Codazzi this implies that

$$
\begin{aligned}
& \nabla_{X}^{\perp} J Y=J \nabla_{X} Y \\
& A_{J X} Y=-J h(X, Y)=A_{J Y} X .
\end{aligned}
$$

The latter formula implies that the cubic form $<h(X, Y), J Z>$ is totally symmetric in all components.

We refer to [1] for the construction of the standard models of indefinite complex space forms $\mathbb{C} P_{s}^{n}(4 \tilde{c})$, when $\tilde{c}>0, \mathbb{C H}_{s}^{n}(4 \tilde{c})$, when $\tilde{c}<0$ and $\mathbb{C}_{s}^{n}$. For our purposes it is sufficient to know that there exist pseudo-Riemannian submersions, called Hopf fibrations,

$$
\Pi: S_{2 s}^{2 n+1}(\tilde{c}) \rightarrow \mathbb{C} P_{s}^{n}(4 \tilde{c}): z \mapsto z \cdot \mathbb{C}^{\star}
$$

if $\tilde{c}>0$ and if $\tilde{c}<0$ by

where

$$
\Pi: H_{2 s+1}^{2 n+1}(\tilde{c}) \rightarrow \mathbb{C} H_{s}^{n}(4 \tilde{c}): z \mapsto z \cdot \mathbb{C}^{\star},
$$

$$
\begin{aligned}
& S_{2 s}^{2 n+1}(\tilde{c})=\left\{z \in \mathbb{C}^{n+1} \mid b_{s, n+1}(z, z)=\frac{1}{\tilde{c}}\right\}, \\
& H_{s+1}^{2 n+1}(\tilde{c})=\left\{z \in \mathbb{C}^{n+1} \mid b_{s+1, n+1}(z, z)=\frac{1}{\tilde{c}}\right\}
\end{aligned}
$$

and $b_{p, q}$ is the standard Hermitian form with index $p$ on $\mathbb{C}^{q}$. For our convenience, we will assume that we have chosen an orthonormal basis such that the first $p$ odd terms appear with a minus sign.

In [1] it is shown that locally any indefinite complex space form is holomorphically isometric to either $\mathbb{C}_{s}^{n}, \mathbb{C} P_{s}^{n}(4 \tilde{c})$, or $\mathbb{C} H_{s}^{n}(4 \tilde{c})$. Remark that, by replacing the metric $<., .>$ by $-<., .>$, we have that $\mathbb{C} H_{s}^{n}(4 \tilde{c})$ is holomorphically anti-isometric with $\mathbb{C} P_{n-s}^{n}(-4 \tilde{c})$. For that purpose, we may assume that $n-2 s \geq 0$ and if $n-2 s=0$, we only need to consider $\mathbb{C}_{s}^{n}$ and $\mathbb{C} P_{s}^{n}(4 \tilde{c})$.

In order to study, or explicitly obtain examples of Lagrangian submanifolds, usually it is more convenient to work with horizontal submanifolds. In that aspect, we first recall some basic facts from [9] which relate Lagrangian submanifolds of respectively $\mathbb{C} P_{s}^{n}(4 \tilde{c})$ and $\mathbb{C} H_{s}^{n}(4 \tilde{c})$ to horizontal immersions in respectively $S_{2 s}^{2 n+1}(\tilde{c})$ and $H_{2 s+1}^{2 n+1}(\tilde{c})$. Here a horizontal immersion $f: M_{s}^{n} \rightarrow S_{2 s}^{2 n+1}(\tilde{c})$ (respectively, $f: M_{s}^{n} \rightarrow H_{2 s+1}^{2 n+1}(\tilde{c})$ ) is an immersion which satisfies if $(p) \perp f_{*}\left(T_{p} M_{s}^{n}\right)$ for all $p \in M_{s}^{n}$, where $\mathrm{i}=\sqrt{-1}$.

Theorem 2.1 ([9]). Let $\tilde{c}>0$ and let $\Pi: S_{2 s}^{2 n+1}(\tilde{c}) \rightarrow \mathbb{C} P_{s}^{n}(4 \tilde{c})$ be the Hopf fibration. If $f: M_{s}^{n} \rightarrow S_{2 s}^{2 n+1}(\tilde{c})$ is a horizontal immersion, then $F=\Pi \circ f: M_{s}^{n} \rightarrow \mathbb{C} P_{s}^{n}(4 \tilde{c})$ is a Lagrangian immersion.

Conversely, let $M_{s}^{n}$ be a simply connected manifold and let $F: M_{s}^{n} \rightarrow \mathbb{C} P_{s}^{n}(4 \tilde{c})$ be a Lagrangian immersion. Then there exist a 1-parameter family of horizontal lifts $f: M_{s}^{n} \rightarrow S_{2 s}^{2 n+1}(\tilde{c})$ such that $F=\Pi \circ f$. Any two such lifts $f_{1}$ and $f_{2}$ are related by $f_{1}=e^{i \theta} f_{2}$, where $\theta$ is a constant. 
The analogous statement for $\tilde{c}<0$ also holds if one replaces $S_{2 s}^{2 n+1}(\tilde{c})$ by $H_{2 s+1}^{2 n+1}(\tilde{c})$ and $\mathbb{C} P_{s}^{n}(4 \tilde{c})$ by $\mathbb{C} H_{s}^{n}(4 \tilde{c})$. Remark that both immersions have the same induced metric and that also the second fundamental forms of both immersions are closely related. For more details see [9].

Next we will introduce the notion of an isotropic submanifold. In Riemannian geometry this was first introduced by $\mathrm{O}^{\prime} \mathrm{Neill}$ in [8]. Isotropic submanifolds of pseudo-Riemannian manifolds were defined in [2]. There a submanifold is said to be (pseudo) isotropic if and only if for every point $p$ of $M$ :

$$
<h(u, u), h(u, u)>=\lambda(p),
$$

does not depend on the choice of $u \in \sum_{p}=\left\{u \in T_{p} M|<u, u\rangle= \pm 1\right\}$. If $\lambda$ is a constant function, the immersion is called constant (pseudo) isotropic.

In the same paper it is also shown that for an immersion to be pseudo isotropic it is sufficient (and equivalent) to work with either spacelike (or timelike) vectors. However it is not equivalent to consider only null vectors. We will also use the following lemma of [2]:

Lemma 2.2. Let $F: M \rightarrow \tilde{M}$ be an isometric pseudo-riemannian immersion. Then the immersion is (pseudo)isotropic if and only if for any tangent vectors $x, y, z, w \in T_{p} M$, we have that

$$
\begin{aligned}
<h(x, y), h(z, w)>+<h(y, z), h(x, w)>+<h(z, x), h(y, w)>=\lambda(p)\{<x, y><z, w>+ \\
<y, z><x, w>+<z, x><y, w>\}
\end{aligned}
$$

\section{Construction of a frame}

Throughout this section we will assume that $M_{1}^{n}$ is a Lagrangian, Lorentzian submanifold of a complex space form $\tilde{M}$. We either will assume that $\tilde{M}=\mathbb{C}_{1}^{n}, \mathbb{C} P_{1}^{n}(4)$ or $\mathbb{C} H^{n}(-4)$ and so we will assume that $c \in\{-1,0,1\}$. We shall also assume that the dimension satisfies $n>2$.

Let $p \in M$. We call a null frame at a point $p$, a frame $\left\{e_{1}, \ldots, e_{n}\right\}$ such that

$$
\begin{aligned}
& <e_{i}, e_{j}>=\left(1-\delta_{i j}\right), \quad i, j \in\{1,2\} \\
& <e_{k}, e_{\ell}>=\delta_{k \ell}, \quad k, \ell \in\{3, \ldots, n\} \\
& <e_{i}, e_{k}>=0, \quad i \in\{1,2\}, \quad k \in\{3, \ldots, n\} .
\end{aligned}
$$

First, we have the following lemma:

Lemma 3.1. If for any null vector $v \in T_{p} M$ we have that $h(v, v)$ is a null vector orthogonal to Jv, then $p$ is a totally geodesic point.

Proof. As $M$ is Lorentzian and Lagrangian, a null vector which is orthogonal to $J v$ is automatically a multiple of $J v$. It follows that

$$
h(v, v)=f(v) J v,
$$

and because of the isotropy condition, see Lemma 2.2, we must have that

$$
<h(v, v), h(v, u)\rangle=\lambda\langle v, v\rangle\langle v, u\rangle=0,
$$

for any $u \in T_{p} M$. Consequently we must have that

$$
0=f(v)<J v, h(v, u)\rangle=f(v)\langle h(v, v), J u\rangle=f(v)^{2}\langle v, u\rangle
$$

Hence $f(v)=0$ and any null vector satisfies $h(v, v)=0$.

We now take an arbitrary null frame at the point $p$ and write

$$
v=e_{1}-\frac{1}{2} \sum_{k=3}^{n} a_{k}^{2} e_{2}+\sum_{k=3}^{n} a_{k} e_{k} .
$$


It is immediate that for any choice of $a_{3}, \ldots, a_{n}$ the vector $v$ is a null vector. So, therefore we must have that

$$
\begin{aligned}
0= & h(v, v) \\
= & h\left(e_{1}, e_{1}\right)+2 \sum_{k=3}^{n} a_{k} h\left(e_{k}, e_{1}\right) \\
& -\sum_{k_{3}}^{n} a_{k}^{2} h\left(e_{1}, e_{2}\right)+\sum_{k, \ell=3}^{n} a_{k} a_{\ell} h\left(e_{k}, e_{\ell}\right) \\
& +\left(\sum_{k=3}^{n} a_{k}^{2}\right) \sum_{k=3}^{n} a_{\ell} h\left(e_{\ell}, e_{2}\right)+\frac{1}{4}\left(\sum_{k=3}^{n} a_{k}^{2}\right)^{2} h\left(e_{2}, e_{2}\right)
\end{aligned}
$$

As the above expression vanishes is a polynomial in $a_{3}, \ldots, a_{n}$ which vanishes identically, it follows that the only now vanishing components of $h$ are

$$
h\left(e_{1}, e_{2}\right)=h\left(e_{3}, e_{3}\right)=\cdots=h\left(e_{n}, e_{n}\right)
$$

As the cubic form is totally symmetric this immediately implies that $p$ is a totally geodesic point.

Therefore, if we now assume that $p$ is not a totally geodesic point, we must have that there exists a null vector $v$ such that $v$ and $A_{J v} v=-J h(v, v)$ are linearly independent. As before, using Lemma 2.2 we have for any vector $u$ that

$$
<A_{J v} v, A_{J v} u>=<h(v, v), h(v, u)>=\lambda<v, v><v, u>=0 .
$$

Taking in particular $v=u$, we get that $A_{J v}$ is a null vector. Moreover, using the fact that $A_{j v}$ is a symmetric operator, we also get that $A_{j v} A_{j v} v=0$. In view of our assumption, if necessary be rescaling $v$ we can now take a null frame such that $e_{1}=v$ and $e_{2}=A_{j v} v$. It immediately follows that

$$
\begin{aligned}
& A_{J e_{1}} e_{1}=e_{2} \\
& A_{J e_{1}} e_{2}=A_{J v} A_{J v} v=0 .
\end{aligned}
$$

showing that the space spanned by $\left\{e_{1}, e_{2}\right\}$ is invariant under $A_{J e_{1}}$. As $A_{J e_{1}}$ is a symmetric operator it follows that the space $\left\{e_{1}, e_{2}\right\}^{\perp}$ is also invariant. As the metric restricted to the latter space is positive definite and the operator is symmetric, we can take orthonormal vectors $\left\{e_{3}, \ldots, e_{k}\right\}$ in this space such that

$$
A_{J e_{1}} e_{k}=\lambda_{k} e_{k}
$$

Then we have:

Lemma 3.2. For all $k \in\{3, \ldots, n\}$, we have that

$$
\lambda_{k}^{3}=-\frac{1}{4} \lambda
$$

Moreover, for all $w \in\left\{e_{1}, e_{2}\right\}^{\perp}$, we have that $h(w, w)=<w, w>\left(-2 \lambda_{3}^{2} J e_{1}+\lambda_{3} J e_{2}\right)$.

Proof. First, we compute $A_{J e_{2}}$ using Lemma 2.2 and the definition of $J e_{2}$. We have for $k, \ell \in\{3, \ldots, n\}$ that

$$
\begin{aligned}
<A_{J e_{2}} e_{k}, e_{\ell}> & =<h\left(e_{k}, e_{\ell}\right), J e_{2}> \\
& =<h\left(e_{k}, e_{\ell}\right), h\left(e_{1}, e_{1}\right)> \\
& =-2<h\left(e_{k}, e_{1}\right), h\left(e_{\ell}, e_{1}\right)> \\
& =-2<A_{J e_{1}} e_{k}, A_{J e_{1}} e_{\ell}> \\
& =-2 \lambda_{k} \lambda_{\ell} \delta_{k \ell} .
\end{aligned}
$$

This implies that

$$
A_{J_{2}} e_{k}=-2 \lambda_{k}^{2} e_{k} .
$$


Hence we see that on $\left\{e_{1}, e_{2}\right\}^{\perp}$ both operators $A_{J_{1}}$ and $A_{J_{e_{2}}}$ are simultaneously diagonalisable. Next we use again Lemma 2.2. We have that

$$
\begin{aligned}
-2 \lambda_{k}^{3} & =<A_{J e_{2}} e_{k}, A_{J e_{1}} e_{k}> \\
& =<h\left(e_{2}, e_{k}\right), h\left(e_{1}, e_{k}\right)> \\
& =\frac{1}{2}\left(\lambda<e_{1}, e_{2}><e_{k}, e_{k}>-<h\left(e_{1}, e_{2}\right), h\left(e_{k}, e_{k}\right)>\right) \\
& =\frac{1}{2} \lambda .
\end{aligned}
$$

Therefore both operators $A_{J e_{1}}$ and $A_{J e_{2}}$ on $\left\{e_{1}, e_{2}\right\}^{\perp}$ are a multiple of the identity. We write $\lambda_{3}=\cdots=\lambda_{n}=\mu$ with $\lambda=-4 \mu^{3}$.

To conclude the proof we now take a unit length vector $w$, orthogonal to both $e_{1}$ and $e_{2}$. Using the previous remarks it follows that the vector

$$
\xi=h(w, w)+2 \mu^{2} J e_{1}-\mu J e_{2}
$$

is orthogonal to both $\mathrm{Je}_{1}$ and $\mathrm{Je}_{2}$. Moreover using again Lemma 2.2 we have that

$$
\begin{aligned}
<\xi, \xi> & =<h(w, w)+2 \mu^{2} J e_{1}-\mu J e_{2}, h(w, w)+2 \mu^{2} J e_{1}-\mu J e_{2}> \\
& =\lambda+4 \mu^{2}<h(w, w), J e_{1}>-2 \mu<h(w, w), J e_{2}>-4 \mu^{3} \\
& =\lambda+4 \mu^{3}+4 \mu^{3}-4 \mu^{3}=0 .
\end{aligned}
$$

Hence it follows that

$$
h(w, w)=-2 \mu^{2} J e_{1}+\mu J e_{2}
$$

which concludes the proof of the lemma.

We now write $\mu=\epsilon \alpha^{2}$, where $\epsilon= \pm 1$ and $\alpha$ is positive. From the previous lemma it follows that $\mu^{3}=-4 \lambda=\epsilon \alpha^{6}$ and thus $\alpha^{3}=\frac{\sqrt{-\epsilon \lambda}}{2}$. We now make the following change of frame, which will change our null frame into a pseudo orthonormal frame. We put

$$
\begin{aligned}
& u_{1}=\frac{1}{2 \alpha}\left(-2 \mu e_{1}+e_{2}\right) \\
& u_{2}=\frac{1}{2 \alpha}\left(2 \mu e_{1}+e_{2}\right) \\
& u_{k}=e_{k},
\end{aligned}
$$

where $k=3, \ldots, n$. In order to check that it is a pseudo orthonormal basis, we only need to compute $<u_{1}, u_{1}>=-<u_{2}, u_{2}>$. Doing so we find that

$$
<u_{1}, u_{1}>=\frac{1}{4 \alpha^{2}}(-4 \mu)=-\epsilon \text {. }
$$


In terms of the new basis we can express the second fundamental form by

$$
\begin{aligned}
A_{J u_{1}} u_{1} & =\frac{1}{4 \alpha^{2}}\left(4 \mu^{2} A_{J e_{1}} e_{1}+A_{J e_{2}} e_{2}\right) \\
& =\frac{1}{4 \alpha^{2}}\left(4 \mu^{2} e_{2}-8 \mu^{3} e_{1}\right) \\
& =\frac{2 \mu^{2}}{\alpha} u_{1} \\
& =2 \alpha^{3} u_{1} \\
A_{J u_{1}} u_{2} & =\frac{1}{4 \alpha^{2}}\left(-4 \mu^{2} A_{J e_{1}} e_{1}+A_{J e_{2}} e_{2}\right) \\
& =-\frac{2 \mu^{2}}{\alpha} u_{2} \\
& =-2 \alpha^{3} u_{2}=A_{J u_{2}} u_{1} \\
A_{J u_{1}} u_{k} & =\frac{1}{2 \alpha}\left(-4 \mu^{2}\right) u_{k}=-2 \alpha^{3} u_{k} \\
A_{J u_{2}} u_{2} & =\frac{1}{4 \alpha^{2}}\left(4 \mu^{2} A_{J e_{1}} e_{1}+A_{J e_{2}} e_{2}\right) \\
& =2 \alpha^{3} u_{1} \\
A_{J u_{2}} u_{k} & =0 \\
A_{J u_{k}} u_{\ell} & =\delta_{k \ell} 2 \alpha \mu u_{1}=2 \epsilon \alpha^{3} \delta_{k \ell}
\end{aligned}
$$

Note that it immediately follows from the above expressions that the mean curvature vector $H(p)$ is in the direction of $J u_{1}$ and that the Lagrangian submanifold is an H-umbilical submanifold in the sense of Chen, which completes the proof of Theorem 1.

From now on we will assume that $M$ is not totally geodesic. Therefore, if necessary by restricting to an open and dense subset we may assume that $\alpha$ is non vanishing. As $n H(p)=2(n-1) \alpha^{3} J u_{1}$ this implies that $\alpha$ is a differentiable function and that $u_{1}$ can be extended to a differentiable vector field on $M$. Therefore, we have shown the following result:

Theorem 3.3. Let $M$ be an isotropic Lorentzian Lagrangian submanifold. Let $p$ be a non totally geodesic point of M. Then in a neighborhood of $p$ there exist pseudo orthonormal vector fields $U_{1}, \ldots, U_{n}$ such that

$$
<U_{1}, U_{1}>=-\epsilon \quad\left\langle U_{2}, U_{2}>=\epsilon \quad<U_{k}, U_{k}>=1,\right.
$$

and such that

$$
\begin{aligned}
h(X, Y)= & 2 \alpha^{3} \epsilon<X, Y>J U_{1}+2<X, U_{1}>\epsilon \alpha^{3} J Y \\
& +2<Y, U_{1}>\epsilon \alpha^{3} J X+8<X, U_{1}><Y, U_{1}>\alpha^{3} J U_{1} .
\end{aligned}
$$

Using the Codazzi equation, we then have

Lemma 3.4. We have that

$$
\begin{aligned}
& X(\alpha)=0, \\
& \nabla_{U_{1}} U_{1}=0, \\
& \nabla_{X} U_{1}=-U_{1}(\log \alpha) X,
\end{aligned}
$$

for any vector field $X$ orthogonal to $U_{1}$.

Proof. From the formula for the second fundamental form we get that

$$
\begin{aligned}
(\nabla h)(Z, X, Y) & =3 Z(\log \alpha) h(X, Y)+2 \alpha^{3} \epsilon<X, Y>J \nabla_{Z} U_{1}+2<X, \nabla_{Z} U_{1}>\epsilon \alpha^{3} J Y \\
& +2<Y, \nabla_{Z} U_{1}>\epsilon \alpha^{3} J X+8<X, \nabla_{Z} U_{1}><Y, U_{1}>\alpha^{3} J U_{1} \\
& +8<X, U_{1}><Y, \nabla_{Z} U_{1}>\alpha^{3} J U_{1}+8<X, U_{1}><Y, U_{1}>\alpha^{3} J \nabla_{Z} U_{1} .
\end{aligned}
$$


By the Codazzi equation we know that

$$
(\nabla h)(Z, X, Y)-(\nabla h)(X, Z, Y)=0 .
$$

First we take $Z, X=Y$ mutually orthogonal and orthogonal to $U_{1}$. We take the component in the direction of $J U_{1}$. Using (3) we get that $Z(\log \alpha) 2 \alpha^{3} \epsilon=0$.

Next we take $Z$ orthogonal to $U_{1}$ and $X=Y=U_{1}$. Using the fact that $Z(\alpha)=0$ and (3) we get from the $J U_{1}$ component of the Codazzi equation that:

$$
0=2<Z, \nabla_{U_{1}} U_{1}>\epsilon \alpha^{3}-8<Z, \nabla_{U_{1}} U_{1}>\epsilon \alpha^{3}
$$

implying that $\nabla_{U_{1}} U_{1}=0$.

Finally, using now the same Codazzi equation, but looking at the general component, we find that

$$
-2 \alpha^{3} J \nabla_{Z} U_{1}+8 \alpha^{3} J \nabla_{Z} U_{1}=3 U_{1}(\log \alpha)\left(-2 \alpha^{3} J Z\right),
$$

which completes the proof.

Using the above lemma we now can describe the pseudo Riemannian structure of $M$ completely. Indeed we have

Theorem 3.5. Let $M$ be an isotropic Lorentzian Lagrangian submanifold. Let $p$ be a non totally geodesic point of $M$. Then in a neighborhood of $p, M$ is locally isometric with a warped product manifold $I \times_{\frac{1}{\alpha}} N$. Moreover, if we denote the standard variable on I by $t$, with $\frac{\partial}{\partial t}=U_{1}$ and $<\frac{\partial}{\partial t}, \frac{\partial}{\partial t}>=-\epsilon$, then $\alpha$ depends only on $t$ and satisfies the following ordinary differential equation:

$$
(\log \alpha)^{\prime \prime}-\left((\log (\alpha))^{\prime}\right)^{2}=-c \epsilon-8 \alpha^{6}
$$

and $N$ is a real space form with constant sectional curvature $\frac{\left.c-4 e \alpha^{6}-\epsilon(\log \alpha)^{\prime}\right)^{2}}{\alpha^{2}}$.

Proof. We define two distribution $T_{1}$ and $T_{2}$ in a neighborhood of $U$. We call $T_{1}=\operatorname{span}\left\{U_{1}\right\}^{\perp}$ and $T_{2}=$ $\operatorname{span}\left\{U_{1}\right\}$. It follows immediately from the previous lemma that both distributions are integrable. We also get that $T_{2}$ is autoparallel. As $\left[Z, U_{1}\right]$ is orthogonal to $U_{1}$ for $Z$ orthogonal to $U_{1}$, it follows also from the previous lemma that $T_{1}$ is spherical with mean curvature normal $\beta E_{1}$, where $\beta=U_{1}(\log \alpha)$. Therefore according to [12], see also [10] we have that $M$ admits locally a warped product structure $M=\mathbb{I} \times_{e f} N$ with $f: I \rightarrow \mathbb{R}$ satisfying

$$
\frac{\partial f}{\partial t}=-\beta=-U_{1}(\log \alpha)
$$

where $f$ and $\alpha$ only depend on the variable $t$, with $\frac{\partial}{\partial t}=U_{1}$. It is clear from the above, that if necessary by applying a homothety to $N$ that we may assume that $e^{f}=\frac{1}{\alpha}$.

Note that by applying the formulas of $\mathrm{O}^{\prime}$ Neill, see Chapter 7 of [7], we get that the curvature tensor $R^{N}$ is given by (taking also into account that the definition of the curvature tensor therein is the opposite of the definition we use here):

$$
R^{N}(X, Y) Z=R(X, Y) Z-\epsilon \frac{1}{\alpha^{2}}\left(\frac{\partial \alpha}{\partial t}\right)^{2}(<Y, Z>X-<X, Z>Y)
$$

where $X, Y, Z$ are vector fields orthogonal to $U_{1}$. By the Gauss equation we have that

$$
\begin{aligned}
<R(X, Y) Z, W>= & c(<Y, Z><X, W>-<X, Z><Y, W>) \\
& \quad+<h(Y, Z), h(X, W)>-<h(X, Z), h(Y, W)> \\
= & \left(c-4 \epsilon \alpha^{6}\right)(<Y, Z><X, W>-<X, Z><Y, W>) .
\end{aligned}
$$


Combining both equations, using the fact that $f=\frac{1}{\alpha}$ it now follows that $N$ is a space of constant sectional curvature $\frac{1}{\alpha^{2}}\left(c-4 \epsilon \alpha^{6}-\epsilon\left((\log \alpha)^{\prime}\right)^{2}\right)$. Note that as this is the sectional curvature of $N$, the above number cannot depend on $t$.

Finally from the Gauss equation, for a vector field $X$ orthogonal to $U_{1}$, we find on the one hand that

$$
\begin{aligned}
<R\left(X, U_{1}\right) U_{1}, X> & =-\epsilon<X, X>c+<h(X, X), h\left(U_{1}, U_{1}\right)>-<h\left(X, U_{1}\right), h\left(X, U_{1}\right)> \\
& =\left(-\epsilon c-8 \alpha^{6}\right) .
\end{aligned}
$$

However using the definition and the previous lemma, we obtain that

$$
\begin{aligned}
<R\left(X, U_{1}\right) U_{1}, X> & =<-\nabla_{U_{1}} \nabla_{X} U_{1}, X>-<\nabla_{\left[X, U_{1}\right]} U_{1}, X> \\
& =<\nabla_{U_{1}} U_{1}(\log \alpha) X, X>+U_{1}(\log \alpha)<\left[X, U_{1}\right], X> \\
& =U_{1}\left(U_{1}(\log \alpha)\right)<X, X>+U_{1}(\log \alpha)<\nabla_{X} U_{1}, X> \\
& =\left(U_{1}\left(U_{1}(\log \alpha)\right)-\left(U_{1}(\log \alpha)\right)^{2}\right)<X, X>.
\end{aligned}
$$

Comparing both expressions now completes the proof of the theorem. Note that the above differential equation could also be obtained by deriving the expression for the sectional curvature of $N$ with respect to $t$. However using that approach we would have needed to consider separately the case when $\alpha$ is constant.

Note that by using a standard existence and uniqueness theorem we could now complete the classification. However, using amongst others the Hopf maps described earlier we will give now a more explicit description of the isotropic submanifolds. Note that the case that $c=0$ follows immediately from the results of [4]. Therefore we will restrict ourselves in the remainder of the paper to the case that $c=-1$ or $c=1$. In both of these cases, we will look at its horizontal lift, denoted by $F$, as described previously.

From the previous theorem we know that there exists a constant $\delta$ such that

$$
c-4 \epsilon \alpha^{6}-\epsilon\left((\log \alpha)^{\prime}\right)^{2}=\delta \alpha^{2} .
$$

This implies that either $c-4 \epsilon \alpha^{6}-\epsilon\left((\log \alpha)^{\prime}\right)^{2}$ vanishes identically on $M$ or it vanishes nowhere on $M$. Note that, if $c=1$ and $\epsilon=-1, \delta$ is necessarily strictly positive. Similarly if $c=-1$ and $\epsilon=1, \delta$ is necessarily strictly negative.

In view of the previous theorem we can introduce coordinates $t$ such that $\frac{\partial}{\partial t}=U_{1}$ and $s_{1}, \ldots, s_{n-1}$ such that $\frac{\partial}{\partial s_{i}}$ belongs to $T_{1}$.

\subsection{Case 1}

We suppose $\delta \neq 0$. We first introduce two vector valued functions $V$ and $W$ by

$$
\begin{aligned}
& \left.V=\left(\frac{\beta}{\alpha}+2 i \alpha^{2}\right) F+\frac{1}{\alpha} U_{1}\right) \\
& W=\left(-\frac{\epsilon c}{\alpha} F+\left(-\frac{\beta}{\alpha}+2 i \alpha^{2}\right) U_{1} .\right.
\end{aligned}
$$

It follows immediately that

$$
\begin{aligned}
& <W, W>=\delta \\
& <V, V>=-\frac{\epsilon \delta}{c}=-\epsilon \delta c \\
& <V, W>=0 \\
& <V, i W>=0 .
\end{aligned}
$$


Denoting by $D$ the covariant derivative of $\mathbb{C}^{n+1}$ it follows from Lemma 3.4 that

$$
\begin{aligned}
D_{X} V & =0 \\
D_{U_{1}} V & =\left(\frac{\beta^{2}}{\alpha}-\frac{c \epsilon}{\alpha}-8 \alpha^{5}-\frac{\beta^{2}}{\alpha}+4 i \alpha^{2} \beta-\frac{-\epsilon c}{\alpha}\right) F \\
& +\left(\frac{\beta}{\alpha}+2 i \alpha^{2}-\frac{\beta}{\alpha}+2 i \alpha^{2}\right) U_{1} \\
& =4 i \alpha^{3} V \\
D_{U_{1}} W & =-2 i \alpha^{3} W \\
D_{X} W & =-\epsilon \delta \alpha W
\end{aligned}
$$

where $X$ belongs to the distribution $T_{1}$.

We introduce now a function $\kappa$ depending only on the variable $t$ by the differential equation

$$
\frac{\partial \kappa}{\partial t}=2 \alpha^{3} .
$$

It now follows that the maps $\tilde{V}=e^{-2 i \kappa} V$ and $\tilde{W}=e^{i \kappa} W$ do not depend anymore on the variable $t$. Hence $e^{-2 i \kappa} V$ is a constant vector of $C^{n+1}$ (with non vanishing length) and $e^{i \kappa} W$ describes a map of the (n-1)dimensional manifold in the (n-1)-dimensional complex hyperplane orthogonal to $e^{-2 i \kappa} V$.

As for $X$ and $Y$ tangent to $T_{1}$, we have that

$$
\begin{aligned}
D_{X} \tilde{W} & =-\epsilon \delta \alpha X e^{i \kappa} \\
D_{X}\left(D_{Y} \tilde{W}\right) & =-\epsilon \delta \alpha e^{i \kappa} D_{X} Y \\
& =-\epsilon \delta \alpha e^{i \kappa}\left(\nabla_{X} Y+2 i \epsilon<X, Y>\alpha^{3} U_{1}-<X, Y>c F\right) \\
& =-\epsilon \delta \alpha e^{i \kappa}\left(\left(\nabla_{X} Y\right)^{T_{1}}+<X, Y>\left(-\epsilon \beta U_{1}+2 i \epsilon \alpha^{3} U_{1}-c F\right)\right) \\
& =d \tilde{W}\left(\left(\nabla_{X} Y\right)^{T_{1}}\right)+\alpha^{2} \delta<X, Y>\tilde{W},
\end{aligned}
$$

where (.. $)^{T_{1}}$ denotes the component of (.) in the direction of the distribution $T_{1}$. From this we see that the $e^{-i \kappa} W$ describes a totally geodesic horizontal submanifold. Moreover, as

$$
F=\frac{1}{\delta \epsilon \alpha}\left(\left(-\beta+2 i \alpha^{3}\right) V-W\right),
$$

we find that after applying an isometry, the immersion is the image after the Hopf map of either

(i) $c=1, \epsilon=-1$ and $\delta=r^{2}$. In this case

$$
F=\frac{1}{\delta \epsilon \alpha}\left(e^{-i \kappa} r y_{1}, \ldots, e^{-i \kappa} r y_{n},\left(-\beta+2 i \alpha^{3}\right) e^{2 i \kappa}\right),
$$

with $-y_{1}^{2}+\cdots+y_{n}^{2}=1$,

(ii) $c=1, \epsilon=1$ and $\delta=r^{2}$. In this case

$$
F=\frac{1}{\delta \epsilon \alpha}\left(\left(-\beta+2 i \alpha^{3}\right) e^{2 i \kappa}, e^{-i \kappa} r y_{1}, \ldots, e^{-i k a p p a} r y_{n}\right),
$$

with $y_{1}^{2}+\cdots+y_{n}^{2}=1$,

(iii) $c=1, \epsilon=1$ and $\delta=-r^{2}$. In this case

$$
F=\frac{1}{\delta \epsilon \alpha}\left(e^{-i \kappa} r y_{1}, \ldots, e^{-i \kappa} r y_{n},\left(-\beta+2 i \alpha^{3}\right) e^{2 i \kappa}\right),
$$

with $-y_{1}^{2}+y_{2}^{2}+\cdots+y_{n}^{2}=-1$,

(iv) $c=-1, \epsilon=1$ and $\delta=-r^{2}$. In this case

$$
F=\frac{1}{\delta \epsilon \alpha}\left(\left(-\beta+2 i \alpha^{3}\right) e^{2 i \kappa}, e^{-i \kappa} r y_{1}, \ldots, e^{-i k a p p a} r y_{n}\right),
$$

with $-y_{1}^{2}+y_{2}^{2}+\cdots+y_{n}^{2}=-1$, 
(v) $c=-1, \epsilon=-1$ and $\delta=-r^{2}$. In this case

$$
F=\frac{1}{\delta \epsilon \alpha}\left(e^{-i \kappa} r y_{1}, \ldots, e^{-i \kappa} r y_{n},\left(-\beta+2 i \alpha^{3}\right) e^{2 i \kappa}\right),
$$

with $-y_{1}^{2}-y_{2}^{2}+y_{3}^{2}+\cdots+y_{n}^{2}=-1$,

(vi) $c=-1, \epsilon=-1$ and $\delta=r^{2}$. In this case

$$
F=\frac{1}{\delta \epsilon \alpha}\left(\left(-\beta+2 i \alpha^{3}\right) e^{2 i \kappa}, e^{-i \kappa} r y_{1}, \ldots, e^{-i k a p p a} r y_{n}\right),
$$

with $-y_{1}^{2}+y_{2}^{2}+\cdots+y_{n}^{2}=1$,

where $\alpha, \beta$ and $\kappa$ are determined by

$$
\begin{aligned}
& \frac{\partial \kappa}{\partial t}=2 \alpha^{3} \\
& \frac{\partial \alpha}{\partial t}=\alpha \beta \\
& \frac{\partial \beta}{\partial t}=\beta^{2}-c \epsilon-8 \alpha^{6},
\end{aligned}
$$

with first integrand given by

$$
c-4 \epsilon \alpha^{6}-\epsilon \beta^{2}=\delta \alpha^{2} .
$$

\subsection{Case 2}

We have $\delta=0$. Note that this case is only possible if $c=\epsilon=-1$ or if $c=\epsilon=1$. As before it follows that $\tilde{W}$ is a constant vector. As it is now a null vector we may assume it is $(1,0, \ldots, 0,1)$. As the manifold $N$, as given in Theorem 4 , is now a flat space we can take local flat coordinates $s_{1}, \ldots, s_{n-1}$. We denote by

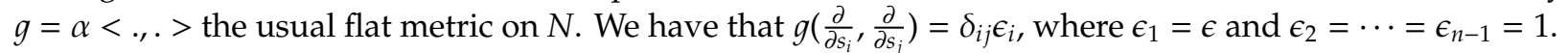

Given that the warping function of the metric is $\frac{1}{\alpha}$, it follows from Theorem 3.3 and Lemma 3.4 we have

$$
\begin{aligned}
F_{t t} & =2 i \alpha^{3} F_{t}+c \epsilon F \\
F_{s_{i} t} & =\left(-\beta-2 i \alpha^{3}\right) F_{s_{i}} \\
F_{s_{i}, s_{j}} & =g\left(\frac{\partial}{\partial s_{i}}, \frac{\partial}{\partial s_{j}}\right) \frac{1}{\alpha^{2}}\left(-\epsilon \beta F_{t}-c F+2 i \alpha^{3} \epsilon F_{t}\right) .
\end{aligned}
$$

Recall that as before

$$
\frac{1}{\alpha^{2}}\left(-\epsilon \beta F_{t}-c F+2 i \alpha^{3} \epsilon F_{t}\right) .=\frac{\epsilon}{\alpha} W(t)=\frac{\epsilon}{\alpha} e^{-i \kappa} \tilde{W}
$$

Integrating the above expression with respect to the variables $s$, taking into account that

$$
\frac{\partial}{\partial t} \frac{e^{-i \kappa}}{\alpha}=\frac{e^{-i \kappa}}{\alpha}\left(-2 i \alpha^{3}-\beta\right),
$$

as well as assuming that at the origin we have the standard basis as initial conditions, it follows that we can write

$$
F\left(t, s_{1}, \ldots, s_{n-1}\right)=\frac{\epsilon}{\alpha}\left(\frac{1}{2} \sum_{j=1}^{n-1} \epsilon_{j} s_{j}^{2}, s_{1}, \ldots, s_{n-1}, \frac{1}{2} \sum_{j=1}^{n-1} \epsilon_{j} s_{j}^{2}\right)+B(t),
$$

where the curve $B(t)$ can be determined in the following way. Using (4) it first follows that

$$
F_{t}=\left(-\beta-2 i \alpha^{3}\right)\left(F+\alpha e^{-i \kappa}(1,0, \ldots, 0,1) .\right.
$$

This gives us that

$$
B^{\prime}(t)=\left(-2 i \alpha^{3}-\beta\right)\left(B(t)+a e^{-i \kappa}(1,0, \ldots, 0,1)\right.
$$


Introducing now a function $\tau$ by the condition that

$$
\frac{\partial}{\partial t} \tau=2 a^{5}
$$

it follows that we can write $B(t)=\left(b_{0}(t), \ldots, b_{n}(t)\right)$, where

$$
\begin{aligned}
& b_{0}(t)=\left(-i \tau-\frac{1}{2} \alpha^{2}\right) \frac{e^{-i \kappa}}{\alpha}+\left(p_{0}+i q_{0}\right) \frac{e^{-i \kappa}}{\alpha} \\
& b_{j}(t)=\left(p_{j}+i q_{j}\right) \frac{e^{-i \kappa}}{\alpha} \\
& b_{n}(t)=\left(-i \tau-\frac{1}{2} \alpha^{2}\right) \frac{e^{-i \kappa}}{\alpha}+\left(p_{n}+i q_{n}\right) \frac{e^{-i \kappa}}{\alpha}
\end{aligned}
$$

The fact that $F$ is contained in a sphere with radius $c$ then implies that $0=p_{1}=\cdots=p_{n-1}, p_{n}=p_{1}+1, q_{n}=q_{1}$ and $p_{1}=1 / 2 \epsilon\left(1+\sum_{j=1}^{n-1} \epsilon_{j} q_{j}^{2}\right)$.

Note that the differential equation for $\alpha$ can be also explicitly solved in this case. It follows that either

$$
\begin{aligned}
& \alpha=\left(\frac{1}{2}\right)^{\frac{1}{3}} \\
& \alpha=\left(1 / 4\left(1-\tanh ^{2}(3 t)\right)\right)^{\frac{1}{6}} .
\end{aligned}
$$

From this the functions $\kappa$ and $\tau$ can also be determined.

\section{References}

[1] M. Barros and A. Romero, Indefinite Kähler manifolds, Math. Ann., 261: 55-62, 1982.

[2] J. L. Cabrerizo, M. Fernández, J. S. Gómez, Rigidity of pseudo-isotropic immersions, J. Geom. Phys., 59: 834-842, 2009.

[3] B. Y. Chen, Complex extensors and Lagrangian submanifolds in complex Euclidean spaces, Tôhoku Mathematical Journal. Second Series, 4: 277-297, 1997.

[4] B. Y. Chen, Complex extensors and Lagrangian submanifolds in indefinite complex Euclidean spaces, Bull. Inst. Math. Acad. Sinica, 31: 151-179, 2003

[5] H. Li, X. Wang, Isotropic Lagrangian submanifolds in complex Euclidean space and complex hyperbolic space Results Math. 56: 387-403, 2009.

[6] S. Montiel, F. Urbano, Isotropic totally real submanifolds, Math.Z., 199: 55-60, 1988.

[7] B. O. Neill, Semi-Riemannian geometry. With applications to relativity, Academic Press, 1983.

[8] B. O. Neill, Isotropic and Kaehler immersions, Canad. J. Math., 17: 907-915, 1965.

[9] H. Reckziegel, Horizontal lifts of isometric immersions into the bundle space of a pseudo-Riemannian submersion, In D. Ferus, R. B. Gardner, S. Helgason, and U. Simon, editors, Global Differential Geometry and Global Analysis 1984. Proceedings of a Conference held in Berlin, June 10-14, 1984, volume 1156 of Lecture notes in Mathematics, pages 264-279. Springer-Verlag, 1984.

[10] H. Reckziegel, R. Ponge, Twisted products in pseudo-Riemannian geometry, Geom. Dedicata, 48: 15-25, 1993.

[11] L. Vrancken, Some remarks on isotropic submanifolds, Publ. Inst. Math. (Beograd), N.S., 51: 94-200, 1992.

[12] H. Wu, On the de Rham composition theorem, Illinois J. math. , 8: 291-311, 1964. 